\title{
Capítulo I \\ El antes y después de las redes \\ sociales en Cuenca
}

Adriel Shicay-Quezada

Camila Villacís-Mendoza

\section{SciELO Books / SciELO Livros / SciELO Libros}

SHICAY-QUEZADA, A., and VILLACÍS-MENDOZA, C. El antes y después de las redes sociales en Cuenca. In: TORRES-TOUKOUMIDIS, Á., and DE SANTIS-PIRAS, A., coord. Los morlacos y las redes sociales [online]. Quito: Editorial Abya-Yala, 2020, pp. 13-32. ISBN: 978-9978-10-534-4. http://doi.org/10.7476/9789978105771.0002.

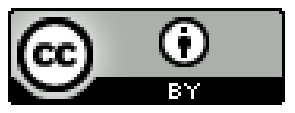

All the contents of this work, except where otherwise noted, is licensed under a Creative Commons Attribution 4.0 International license. Todo o conteúdo deste trabalho, exceto quando houver ressalva, é publicado sob a licença Creative Commons Atribição 4.0. Todo el contenido de esta obra, excepto donde se indique lo contrario, está bajo licencia de la licencia Creative Commons Reconocimento 4.0. 


\title{
CAPítulo I El antes y después de las redes sociales en Cuenca
}

\author{
Adriel Shicay-Quezada \\ Universidad Politécnica Salesiana, Ecuador \\ https//orcid.org/0000-0001-9218-3200 \\ Camila Villacís-Mendoza \\ Universidad Politécnica Salesiana, Ecuador \\ https://orcid.org/0000-0002-1364-8290
}

\section{Resumen}

El impacto comunicacional que ha generado las redes sociales en la vida de las personas es una verdad indiscutible de nuestros días; su uso progresivo a la par de los avances tecnológicos y de comunicaciones, las ha convertido en una herramienta clave para los procesos comunicativos y sociales. Pero, ¿cómo ha sido esta transición a la comunicación virtual de la sociedad cuencana en los últimos años? El objetivo de este estudio es analizar las formas de comunicación interpersonal mediante la red social Fa- 
cebook y observar los efectos que ha tenido en las relaciones sociales en Cuenca en comparación con los antiguos medios de comunicación tradicionales. Las preguntas de esta investigación se respondieron a través de entrevistas a profundidad a 30 residentes de Cuenca, Ecuador mediante plataformas en línea. Las respuestas obtenidas demuestran la cantidad de usuarios activos de esta red social que se encuentran satisfechos con sus facilidades, su aprovechamiento para el acceso y difusión de la información tanto para promocionar y publicitar sus negocios, como para informarse sobre noticias de actualidad. Se comprendió también la aceptación que tiene la plataforma dentro de la sociedad considerando la opinión de la muestra en lo referente a si la integración de redes sociales ha mejorado o empeorado las relaciones sociales en la población de Cuenca. Estos resultados indicaron el cambio comunicacional que ha tenido el paso de redes sociales en la ciudad y las diferencias más destacables entre el antes y después de su inmersión en la vida cotidiana.

Palabras clave: Cuenca, Facebook, red social, comunicacional, relaciones sociales, usuarios, cuencanos, comunicación interpersonal.

\section{Abstract}

The communicational impact that social networks have generated in people's lives is an indisputable truth of our times; their progressive use, along with technological and communication advances, has made them a key tool for communicative and social processes. But, how has 
this transition to the virtual communication in the society of Cuenca occurred in the last years? The purpose of this study is to analyze the forms of interpersonal communication through the social network Facebook and to observe the effects it has had on social interactions in Cuenca in comparison with the former traditional methods of communication. The questions in this investigation were responded to through in-depth interviews with 30 residents of Cuenca, Ecuador using online platforms. The answers obtained show the number of active users of this social network who are satisfied with its facilities, its use for access and spreading of information in order to promote and advertise their businesses, and to stay informed about current news. The answers collected contributed to a better understanding of the acceptance of the platform within society, considering the opinion of the sample as to whether the integration of social networks has improved or worsened social relations in the population of Cuenca. These results indicated the communicational change that the passing of social networks in the city has had and the most remarkable differences between before and after its immersion in daily life.

Keywords: Cuenca, Facebook, social network, communication, social relations, users, interpersonal communication.

El impacto de las redes sociales y su inmersión en nuestra vida cotidiana es, sin duda, una realidad innegable a nivel global en nuestros días. El nacimiento del internet se sitúa en la década de 1960, en el contexto contemporáneo de la Guerra Fría, como una herramienta militar de los 
Estados Unidos; pero no fue hasta 20 años después cuando revolucionó al mundo con su libre comercialización. Sin embargo, la llegada de la "red" a Cuenca, Ecuador se hizo esperar hasta mediados de los años 90 y se popularizó a inicios del nuevo milenio.

La historia de cómo la web y las redes sociales se insertaron en el marco cultural de nuestra sociedad es una amplia fuente de análisis; no obstante, el objetivo de esta investigación no se basa únicamente en una narrativa lineal de la evolución de las redes sociales en la Ciudad de los Cuatro Ríos, sino más bien en contrastar su impacto y la influencia que ha tenido en los ámbitos de la comunicación personal y social, antes y después de su incorporación en realidad colectiva cuencana.

Cuenca es la tercera ciudad más grande en población ecuatoriana, con un total de 505585 habitantes según expone el Instituto Nacional de Estadística y Censos (INEC, 2020). A través de una indagación realizada en 2015 y publicada por el Diario El Comercio, se demostró que: "A escala nacional, un 41,4\% de las personas encuestadas tienen una cuenta en una red social. Entre las opciones presentadas por el INEC están Facebook, Twitter, YouTube, WhatsApp, Skype y otras redes" (Sandoval, 2015).

En la era de la digitalización, existen tres principales factores que moldean las relaciones de adaptación entre las redes sociales y los actores de la sociedad: componer, compartir y difundir (García-Galera \& Valdivia, 2014). 


\section{7}

Este primer componente permite a los usuarios ser partícipes activos de la interacción y publicación de contenido dentro de la red; las posibilidades y la facilidad de producir sobrepasan las limitaciones de generaciones pasadas; haciendo sencillo para el internauta convertirse en "prosumidor", concepto desarrollado por el profesor de literatura Marshall Herbert McLuhan en 1972.

El prosumo tiene su esencia en la unión de dos conceptos: productor y consumidor. Al principio no se refería al campo tecnológico sino al campo económico ya que señalaba al prosumidor a aquella persona que elaboraba sus propios productos y los consumía, con la ausencia de intermediarios. (McLuhan \& Nevitt en Arribas-Urrutia et al., 2019, p. 52)

El concepto de compartir información se basa en la asequibilidad de las redes sociales "al alcance de todos". Cualquier individuo es capaz de generar y compartir contenido a través de sus redes sociales, fin fundamental de esta acción: "Crear sin compartir no tiene sentido" (García-Galera \& Valdivia, 2014). Es gracias a la sencillez de este proceso, como surge el término "periodismo móvil" que consiste en la utilización de un dispositivo portátil para redactar o filmar sucesos y luego compartirlos en redes sociales, hecho que en el contexto social cuencano se ha visto presente varias veces en sucesos político-sociales, donde debido al quebrantamiento de la confianza del pueblo con la prensa nacional, este personaje, el ciberperiodista, adquirió veracidad. "El periodismo no muere, muta" dijo la autora Carmela Ríos en 2012 en la entrega del Premio Ortega y Gasset. 
En referencia al factor de difusión, las redes sociales permiten llegar a un público más amplio sin límites de tiempo y espacio, son útiles para crear relaciones interpersonales entre interesados sobre un tema en común; debido a esto, en el marketing, cumplen un rol fundamental para la divulgación publicitaria.

La relación entre la sociedad y las redes sociales, no es estática, se encuentra en constante evolución, razón por la cual las empresas de nuestro medio se han visto en la necesidad de adaptarse para satisfacer el nuevo estilo de los demandantes, en particular los emergentes microemprendimientos han visto en esta disponibilidad de fácil difusión una oportunidad para hacer conocer sus productos y servicios, logrando llegar a un mayor público en menor tiempo y así acelerando su crecimiento. Por tanto, es posible afirmar que las redes sociales y las aplicaciones móviles han consolidado una relación bilateral más cercana entre productor y consumidor mediante la retroalimentación o feedback (Aucay \& Herrera, 2017).

\section{Metodología}

Objetivo general: Analizar las relaciones de comunicación interpersonal antes y después de la incorporación de las redes sociales, en Cuenca, Ecuador.

Objetivos específicos: Observar las formas de interacción interpersonal en redes sociales, específicamente en la plataforma Facebook; Comparar las relaciones de comunicación interpersonal antes y después las redes so- 
ciales como parte de la cotidianeidad de la sociedad cuencana, tomando como referencia la plataforma Facebook; Contrastar el cambio comunicacional en las relaciones interpersonales, influenciadas por redes sociales, tomando como referencia las plataformas Facebook en Cuenca, con relación a los medios tradicionales de comunicación.

\section{Enfoque}

Esta investigación tiene un enfoque cualitativo al estudiar las características del paso de las redes sociales en la sociedad cuencana. Cuenta con un análisis deductivo partiendo de las generalidades de las redes sociales a nivel histórico hacia la particularidad de los efectos de las mismas en las relaciones interpersonales en Cuenca.

\section{Diseño}

Este proyecto está basado en un diseño narrativo al exponer nociones generales sobre las redes sociales y el impacto de las mismas, a través de conceptos previos presentados por autores nacionales e internacionales; con la finalidad de contrastar las características de la comunicación interpersonal, relatando el antes y después de la introducción de las redes sociales en la comunidad cuencana, mediante documentación y entrevistas en profundidad.

\section{Herramientas de recopilación de datos}

Las herramientas a utilizar en el desarrollo de este estudio son entrevistas a profundidad de opinión, expre- 


\section{0}

sión de sentimientos y especialmente de antecedentes, pues buscamos contrastar y observar los cambios que han provocado las redes sociales en las relaciones interpersonales en los ciudadanos cuencanos. El formato de las preguntas será: general para crear un ambiente de confianza con el entrevistado, de ejemplificación, estructurales y de contraste. La segunda técnica de recolección de datos se cimenta en documentos, materiales y registros al revisar artículos e investigaciones previas sobre el tema a trabajar.

\section{Criterios de análisis}

\section{Viabilidad de medios comunicativos:}

- Canales para la comunicación: Forma en que los individuos efectúan distintos procesos comunicativos, indispensablemente de la intención del mensaje, a través de un canal. Los canales pueden ser abstractos o concretos, es decir, digitales (televisión, teléfono, radio, portales de medios electrónicos, etc.) o físicos (Periódicos, diarios, cartas, etc.)

- Medios de comunicación tradicionales: Televisión, radio, prensa escrita, llamadas telefónicas convencionales, Mensajes de texto, correo electrónico, entre otros.)

Inversión y distribución de tiempo: Desde las expresiones de la muestra seleccionada se realiza un cuestionamiento al tiempo desde que pasaron a formar parte de la digitalidad, la cantidad de tiempo que estos invierten para 


\section{1}

el uso de las redes sociales, específicamente Facebook, y la forma en cómo se organizan con el resto de actividades sistemáticas de su cotidianidad para crear un espacio dedicado al uso y consumo de la virtualidad, que ofrecen este tipo de redes sociales.

Percepción del aprovechamiento comunicacional: Juicios de valor emitidos en referencia a la factibilidad del uso de redes sociales como herramienta de procesos comunicativos, así como también las sensaciones o sentimientos, denotados en las versiones de los entrevistados en cuanto al aprovechamiento funcional de la red social.

\section{Resultados}

A continuación, presentamos nuestros resultados como parte esencial de nuestro capítulo, mediante la interpretación de las opiniones vertidas por los entrevistados, que corresponden a la muestra; apoyados en gráficos estadísticos que están relacionados a los criterios de análisis planteados en nuestra metodología. Es importante mencionar que en algunos casos hemos redactado textualmente opiniones cortas, emitidas por los entrevistados, como datos extras que caben ser destacados en nuestra investigación.

\section{Viabilidad de medios comunicativos}

Los entrevistados declararon que, las llamadas mediante un teléfono convencional o celular eran las más utilizadas al momento de comunicarse con los demás (38\%), 


\section{2}

seguido por los mensajes de texto pagados (29\%), luego estaban otras redes sociales como MySpace, Hi5, Windows Live Messenger, entre otros (19\%). Mientras que, de manera personal a través de la oralidad, las personas también se comunicaban, pero de una forma menos frecuente (10\%), y finalmente, los correos electrónicos, que, a pesar de ser una vía de comunicación virtual, eran los menos usados (4\%).

\section{Figura 1}

Cambio generado por Facebook al momento de comunicarse con el círculo social

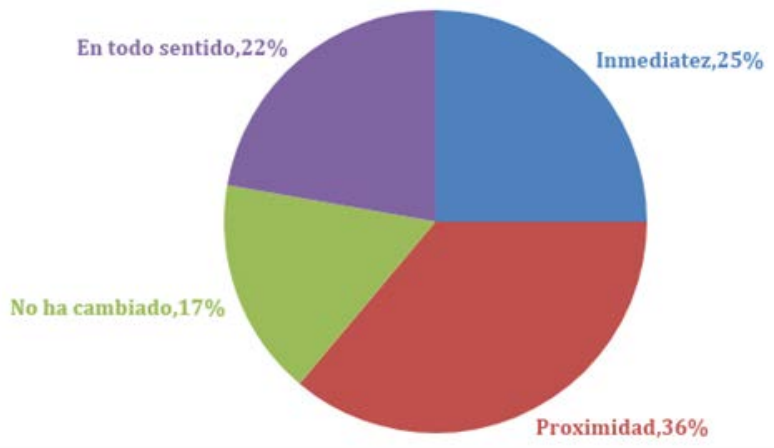

Fuente: Elaboración propia.

La proximidad ha sido la característica más nombrada al momento de evidenciar el cambio comunicacional por el uso de Facebook (36\%), seguido por la inmediatez $(25 \%)$ que no difiere mucho en cuanto a las personas que piensan que, simplemente ha cambiado en todo sentido (22\%); para finalmente referir una cantidad considerable, y no menos importante, de quienes piensan que no ha cambiado su manera de comunicarse (17\%). 


\section{3}

Ha facilitado la comunicación sin barreras físicas, nos ha permitido estar al tanto de nuestro entorno incluso de manera informativa, pero también ha perjudicado las relaciones interpersonales con el entorno, además ha dado paso al cyberbullying y la exposición de información personal (Anónimo).

\section{Figura 2}

Medios de información que, según los entrevistados, han sido desplazados por la aparición de Facebook

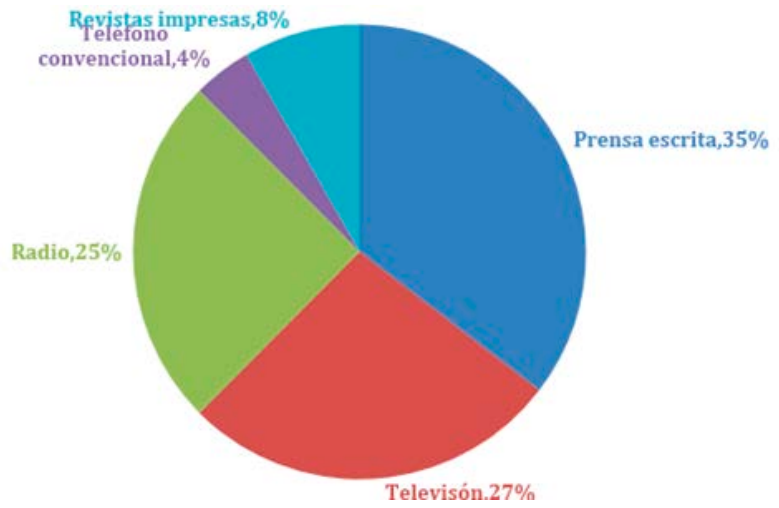

Fuente: Elaboración propia.

Si hay algo en que gran parte de los entrevistados están de acuerdo es que, varios medios de comunicación/ información han sido desplazados por Facebook por sus distintas características. Así es el caso de la prensa escrita (diarios y periódicos), que se posiciona como el medio que primero ha sido desplazado por esta red social al momento de informar (36\%), seguido por la televisión que a pesar de tener una eficaz manera de transmitir mensajes a 


\section{4}

través de material audiovisual, lo posicionan como el segundo medio desplazado (27\%), luego está la radio, que siendo un medio tradicional, aún se mantiene en pie por el consumo de grupos sociales específicos (25\%), ocupan el cuarto lugar de desplazamiento las revistas impresas, los entrevistados consideraron que no es una buena manera de comunicar un mensaje; sin embargo, no formó parte ni siquiera de los tres primeros medios desplazados por Facebook $(8 \%)$ y por último está el teléfono convencional, medio que aún se usa para una comunicación no constante (4\%). Adicional a esto, las personas que cumplieron con la entrevista expresan que, los medios convencionales no desaparecerán fácilmente, como es el caso de la radio, que, por su facilidad de sintonización en distintos lugares, ha permanecido en actividad y con una acogida considerable.

\section{Inversión y distribución de tiempo}

La mayoría de los entrevistados indicaron que se unieron como usuarios de Facebook con una cuenta activa, desde hace seis a diez años (55\%), seguido por otra representativa cantidad que lo hicieron en un intervalo de once a quince años (41\%), y finalmente una cantidad pequeña que refiere hace uno a cinco años (4\%).

Es decir que, Facebook mantiene actividad desde que llegó al Ecuador hace quince años, pero hace diez años es donde registra mayor acogida por parte de usuarios cuencanos. 


\section{5}

\section{Figura 3}

Tiempo desde que la persona se unió a Facebook y lo usa de manera activa

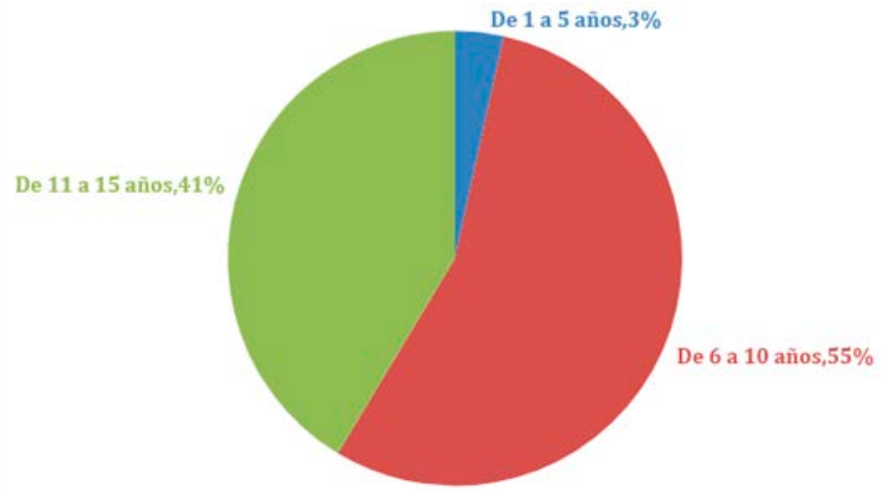

Fuente: Elaboración propia.

Figura 4

Número de horas invertidas en el uso

de la red social Facebook

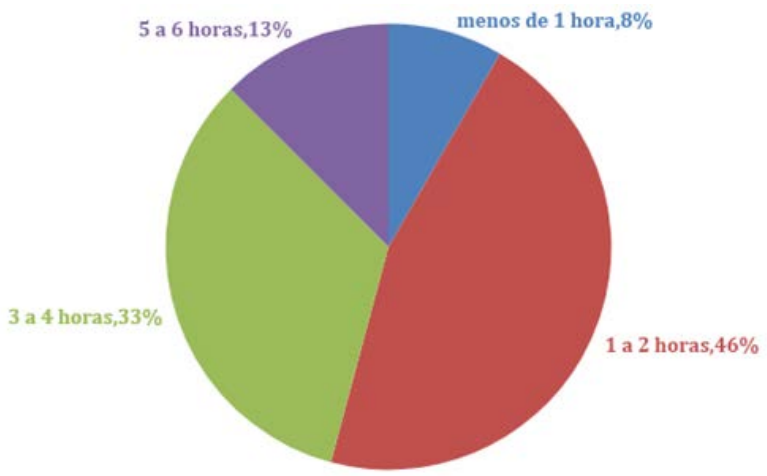

Fuente: Elaboración propia. 


\section{6}

Gran parte de los usuarios de Facebook en Cuenca dijeron que ocupan alrededor de una a dos horas en el uso de esta red social (46\%), seguido por otros que gastan de tres a cuatro horas al navegar en esta plataforma web (33\%), luego en tercer lugar, están los usuarios que ocupan alrededor de cinco a seis horas (13\%); y finalmente están quienes se toman menos de una hora para revisarlo $(8 \%)$.

Algunos individuos que se ubican en aquel grupo que consumen Facebook durante una a dos horas, expresaron que lo hacen durante la noche, dato que nos pareció importante destacar.

\section{Figura 5}

Manera en que Facebook ha vulnerado la distribución del tiempo destinado a la realización de actividades diarias

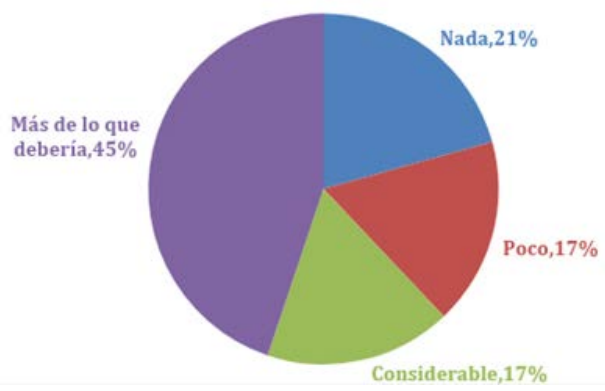

Fuente: Elaboración propia.

En el análisis de este criterio hemos encontrado que la mayoría de la gente (45\%) piensa que ha cambiado mucho la manera de distribuir su tiempo, al otorgar más 
tiempo de lo debido, seguido de quienes piensan que no ha cambiado para nada al usarlo (21\%), y finalmente están quienes dijeron que, es poco y considerable, la manera en que ha cambiado la manera en que distribuían su tiempo por usar Facebook (17\%).

\section{Percepción del aprovechamiento comunicacional}

\section{Figura 6}

La facilidad que Facebook ha proporcionado a la muestra entrevistada, misma que antes no poseían sino hasta su uso

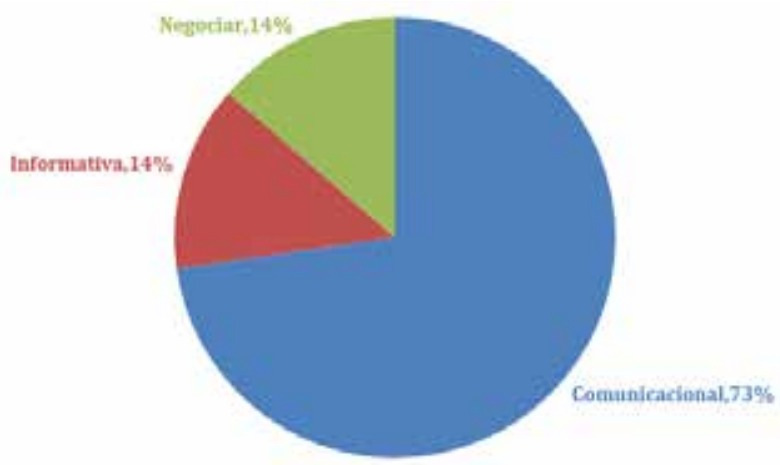

Fuente: Elaboración propia.

La mayoría de las personas entrevistadas supieron indicar que Facebook mayormente brinda una facilidad comunicacional al momento de estar en contacto y pendientes de lo que sucede en las vidas de sus conocidos (73\%), seguida por la facilidad de hacer negocios a través de pequeños y grandes emprendimientos difundidos por 
medio de esta red social (14\%); luego que la facilidad informativa no se queda atrás y se la efectúa por parte de un cierto público considerable (13\%).

Respecto a la obtención de información de actualidad a través de Facebook, a comparación de los medios tradicionales (radio, televisión y prensa escrita), varias personas dicen sentirse satisfechos, ya sea por su poca disponibilidad de tiempo para informarse y poder hacerlo mientras se comunican con terceros, así también por tener mayor dinamismo en contenido multimedia.

\section{Figura 7}

Expresiones del efecto que tiene Facebook en las relaciones de comunicación interpersonal en Cuenca

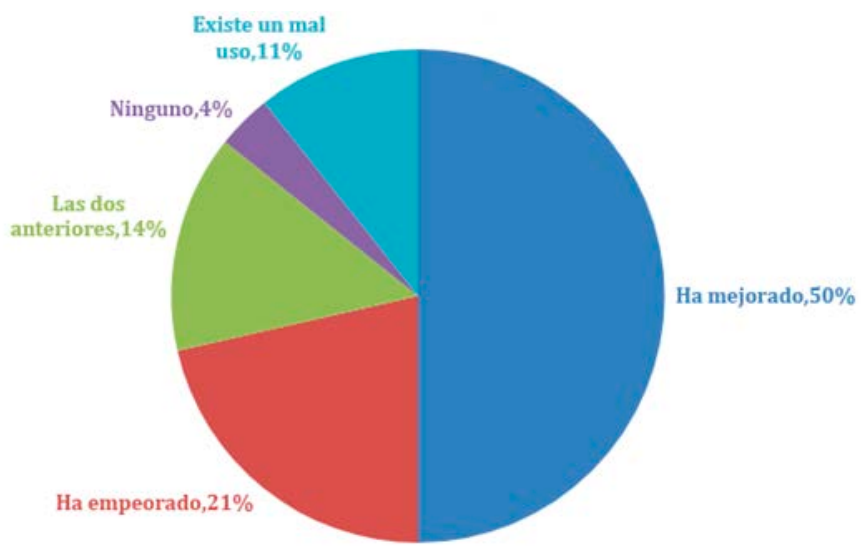

Fuente: Elaboración propia.

Por otra parte, pocas personas están conscientes y sienten preocupación al saber que existe la posibilidad de 
encontrarse con información falsa o distorsionada, que únicamente genera confusión y desinformación.

Finalmente, una parte minúscula de la muestra expresó que no le causa ninguna emoción, ya que no se informan a través de Facebook.

La mitad de nuestros entrevistados han sabido reconocer que Facebook ha mejorado el nivel de comunicación interpersonal existente en Cuenca (50\%), mientras que una quinta parte ha dicho lo contrario (21\%), seguido de otra cantidad pequeña la cual expresó que tiene aspectos positivos y negativos (14\%), mientras que una décima parte señala un aspecto importante como lo es el mal uso que se le da a esta red social (11\%); y finalmente una minúscula cantidad de personas que piensa que no ha tenido ningún efecto (4\%).

Una de las expresiones que vale exponerla es la siguiente: "Si bien es cierto, las redes sociales acercaron a los que están lejos, pero es cierto que también han alejado a los que están cerca, porque existe una preferencia hacia la tecnología antes que a lo tradicional".

\section{Conclusiones}

La comunicación es un proceso que se encuentra en constante evolución, se adapta a los avances tecnológicos, a los cambios sociales y variaciones culturales. Tras un profundo análisis de los estudios presentados, y en base a los objetivos propuestos, se logró comprender las formas en que las redes sociales, tomando como referencia Facebook, 
han moldeado las relaciones interpersonales en Cuenca en los últimos años.

Mediante las entrevistas en profundidad se observó la aceptación de la generalidad de la población hacia la plataforma como herramienta de uso diario, debido a sus facilidades comunicativas, su simplicidad de manejo y su versatilidad hacia diversas actividades de interés de los entrevistados, como: el acceso a noticias, información, publicidad, actualidad de sus contactos y del mundo. Sin embargo, es conveniente mencionar que un considerable porcentaje de la muestra encontró que la red social "ha empeorado las relaciones interpersonales" en la ciudad de estudio, además que reconocieron el mal uso de esta plataforma y la existencia de falsa información dentro de la misma.

El estudio previamente presentado permitió observar, analizar y comparar las relaciones de comunicación interpersonal antes y después la inmersión de las redes sociales como parte de la cotidianeidad de la sociedad cuencana.

Gran parte de las preguntas de la entrevista fueron de opinión, por lo tanto, inferimos también en que las respuestas de la presente investigación proporcionan resultados regidos a la subjetividad y percepción personal de cada uno de los entrevistados. Para mayor contraste de datos, es recomendable para futuros investigadores considerar una mayor cantidad de personas dentro de la muestra a analizar. Esta indagación se realizó en el periodo mayo-julio, 2020; en contemporaneidad con la pandemia Covid-19 y las medidas de distanciamiento social implementadas por 
el gobierno ecuatoriano, razón por la cual se alcanzó una menor cercanía de diálogo con la muestra entrevistada.

\section{Bibliografía}

Arribas-Urrutia, A., Islas-Carmona, O., \& Cortés, F.G. (2019). From prosumers to observers: an emergent trend on the Internet and among Young Ecuadorians. Results from the World Internet Project study, Ecuador. Revista Latina de Comunicación Social, (74), 969-996. http:// dx.doi.org/10.4185/RLCS-2019-1367

Aucay-Piedra, E., \& Herrera-Torres, P. (2017). Nivel de uso de las redes sociales en el proceso de comunicación en las MIPES de Cuenca. Retos. Revista de Ciencias de la Administración y Economía, 7(14), 81-98. http://dx.doi. org/10.17163/ret.n14.2017.04

García- Galera, \& Valdivia, A. (2014). Prosumidores mediáticos: Cultura participativa de las audiencias y responsabilidad de los medios. Comunicar: Revista científica de Comunicación y Educación, (43), 10-13. https://bit. ly/3hbMoy 2

INEC (2020). Instituto Nacional de Estadística y Censos. https:// bit.ly/3hlrH2M

Sandoval, C. (15 de abril de 2015). El Comercio. https://bit. ly/2YkNHUa

\section{Anexos}

Cuestionario para la aplicación de las entrevistas:

1. ¿Hace cuánto tiempo se unió a la red social Facebook?

2. ¿Cómo se comunicaba con su círculo social antes del uso de Facebook? 
3. ¿Cómo considera usted que Facebook ha cambiado la manera de comunicarse con su círculo social?

4. ¿Cuál cree que es la facilidad que le ha proporcionado Facebook, misma que no poseía antes del uso de esta red social?

5. ¿Cuánto tiempo invierte diariamente en Facebook? (subir contenido, compartir, comunicarse con sus contactos)

6. ¿De qué manera piensa usted que Facebook ha cambiado la distribución del tiempo que invierte en diferentes actividades de su día a día?

7. ¿Cómo se siente usted cuando obtiene información de actualidad a través de Facebook a comparación de los medios tradicionales (radio, tv, prensa escrita)?

8. ¿Qué medios de información considera usted que han sido desplazados por Facebook?

9. Desde su punto de vista ¿Las redes sociales, específicamente Facebook, han mejorado o empeorado las relaciones de comunicación interpersonal en Cuenca? 